\title{
Cooperative Power Consumption in the Smart Grid Based on Coalition Formation Game
}

\author{
Xi Luan*, Jianjun Wu*, Shubo Ren*, Haige Xiang* \\ *Institution of Advanced Communications, EECS, Peking University, China \\ Email: luanxi@pku.edu.cn, just@pku.edu.cn
}

\begin{abstract}
The power consumption schemes of consumers is an important issue in energy management process in smart grid. The non-cooperative methods which are always considered cannot achieve the maximized performance for consumers and networks. In this paper, we propose a cooperative power consumption scheme for consumers based on coalition formation game, which is suitable for the general electricity markets in smart grid. The advantage is that it can utilize the cooperative relationships among each other for payment savings and meanwhile take the social welfare into consideration. It is realized according to the pricing model used by power provider, the welfare function of the consumer coalitions, as well as the coalition formation algorithm based on the modified Pareto order which are proposed in this paper. Simulation results show that a stable consumers' partition can be formed in the concerned area and the higher utility for consumers and social welfare can be obtained comparing with the non-cooperative methods.
\end{abstract}

Keywords-Smart Grid, Demand-side management, Coalition formation, Pricing model, Modified Pareto order

\section{INTRODUCTION}

Comparing with traditional power grid, smart grid has many advantages. For example, it is a robust, flexible and efficient distributed power network in which both consumers and energy providers all have more choices [1]. Though more advantages are obtained in smart grid, more complex energy management schemes, which always refers to demand-side management (DSM), are also brought in. In the DSM process, the power consumption strategies of consumers play an important role which not only impact the level of their own satisfaction, but also influence the benefits and performance of the whole network.

Recently, the DSM as well as the consumers behaviours draw more and more attention in research area. To ensure the stabilization and efficiency of smart grid, several DSM methods have been proposed, such as time of use (TOU), critical peak pricing (CPP), or Real-time pricing (RTP) [2]. From the behaviour type of consumers, the exist management methods can be classified as non-cooperative and cooperative ones. In non-cooperative methods, non-cooperative games are always considered with different purposes [3] [4]. Though it is simple and there is no need for information exchange process among consumers, the maximized utilities for consumers and the optimal social welfare for the networks cannot be always achieved. If cooperations between consumers are permitted, more benefits can be obtained correspondingly, such as the centralized optimization scheme [5]. However, it requires perfect information sharing among all consumers or a central controller to collect consumers information to make decision, and therefore not practical for large-scale networks. In [6] and [7], the concept of coalition formation among energy consumers are introduced as compromises. However, the scheme in [6] are only suitable for some European countries who have two different electricity markets and there is no feasible algorithm for coalition formation in [7].

In this paper, we propose a cooperative power consumption scheme for consumers in smart grid, which is suitable for general electricity markets. First, a two parts pricing model and the corresponding welfare function of the consumer coalitions are proposed. In this system, wireless communication is used among consumers as Wireless mesh network (WMN). After that, a modified Pareto order is defined as a comparison relation for different partitions. It not only performs the comparison using the payoff of individual consumers but also the social welfare in network as well. Then, a coalition formation algorithm according to the modified Pareto order is devised for the consumers in smart grid. It is based on merge and split rules. At last, the efficiency of the proposed scheme are verified by simulation.

This paper is organized as follows: The network and pricing model is introduced in Section II. Then, we formulate the problem as a coalition formation game in Section III. In Section IV, an algorithm for coalition formation is devised. Simulation results are presented in Section V. Finally, conclusions are drawn in Section VI.

\section{System ModeL}

In the section, we present the network model at first. Then, the pricing model and the cost for information exchange are given.

\section{A. Network Model}

Consider a smart power system with power generation side and power consumption side. At the power generation side, all distributed power providers are seen as a single entity since the key issues is not the distributed power generators in this paper. At the power consumption side, all consumers in the concerned area (for example, houses in several communities) are denoted by the set $\mathcal{N}$, where $N \triangleq|\mathcal{N}|$ is the number of consumers. An illustration of the network model is shown in Fig. 1. In each house, a smart meter is equipped as an agent, which decides 


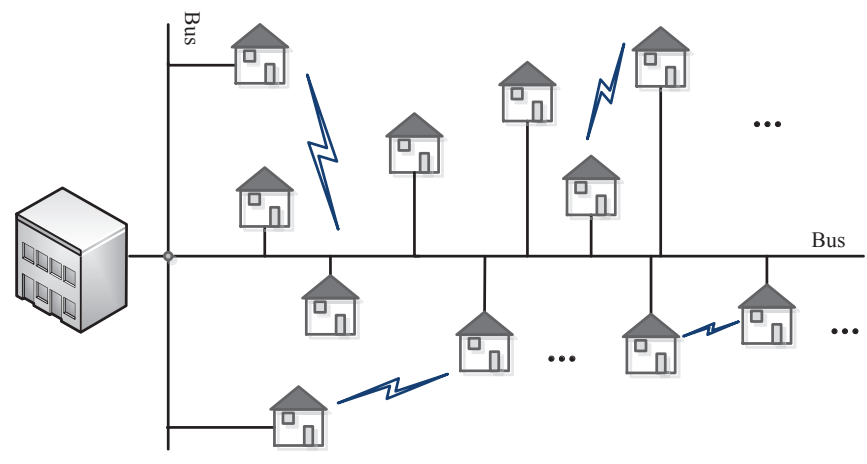

Fig. 1. An simplified graph demonstrates the network model in smart grid.

the power consumption of the consumer intelligently. The intended operation time of consumers can be divided into $T$ time periods, where $T \triangleq|\mathcal{T}|, \mathcal{T}$ is the set of all time periods. In the $t$ th time period, we define the power consumption vector of the $i$ th consumer as $\mathbf{x}_{i}=\left[\begin{array}{lllll}x_{i}^{1} & \cdots & x_{i}^{t} & \cdots & x_{i}^{T}\end{array}\right]$, where $x_{i}^{t}$ denote the power consumption of the $i$ consumer in the $T$ time period. Considering the actual situation, the minimum and maximum power consumption for each user should be restricted, that is

$$
0 \leq x_{i}^{t} \leq x_{\max }, \forall t \in \mathcal{T},
$$

Therefore, the total power demand in the time period is

$$
P_{t}=\sum_{i \in \mathcal{N}} e_{i, t}, \forall t \in \mathcal{T}
$$

\section{B. Pricing Model}

Take into account the cost of power generation and power load profiles, power provider can set a smart pricing for demand-side management. In this paper, the price contains two part: the wholesale price $\phi_{t}$ and the price gap $\eta_{t}$ [8]. Therefore, the price scheme by power provider in this paper is $p_{t}=\phi_{t}+\eta_{t}$.

The first part of price is set according to the cost for power generation, which is $\phi_{t}=C\left(P_{t}\right) / P_{t}$. The cost function $C\left(P_{t}\right)$ should be monotonously increasing to $P_{t}$, since more fuel and sources are needed to generate more energy. We choose a quadratic cost function in this paper, such as $C\left(P_{t}\right)=\alpha P_{t}^{2}+\beta+\lambda$, where $\alpha>0, \beta \geqslant 0$ and $\lambda \geqslant 0$ are the fixed coefficients [9]. The second part of smart price is influenced by the variation of power demand and target power profiles, which is designed as following,

$$
\eta_{t}=\mu\left(P_{t}-P_{v}\right)^{2},
$$

where $P_{v}$ is the target average power in each time period and $\mu>0$ is the demand impact factor. From the price gap defined above we can see that it is a strictly convex function. If the power consumption is less than $P_{v}$, the price gap will decrease and become incentive price to motivate consumers buy more electricity, consequently more benefits for power provider. On the contrary, too much energy request will increase the price because of the heavy load in power line.

Based on this smart pricing model, consumers can schedule their power consumption. In non-cooperative game method, each distributed consumer decides their own power demand independently. Though it is simple and there is no need for information exchange, the maximized utilities cannot be always achieved.

\section{Information Exchange}

In this paper, we devise a cooperative strategy for consumers in smart grid. All consumers participate in the coalition formation game to find their appropriate partner to maximize their utilities. However, in order to realize cooperation among consumers, information exchange is necessary. In this process, the smart meter belonging to a certain consumer broadcasts information in wireless mode with a portion of the available power. Let $\hat{i}$ denote the index of the farthest smart meter which is to be communicated by the smart meter $i$. According to the broadcast nature, all the other ones in this scope can also obtain the information. The power loss of the $i$ th smart meter is [10]

$$
L_{i, \hat{i}}=\gamma \sigma^{2} d_{i, \hat{i}}^{\omega}
$$

where $\sigma^{2}$ is the noise variance, $\gamma$ is the target average SNR for information exchange. $d_{i, \hat{i}}$ denotes the distance between the $i$ th smart meter and the farthest one it desires to be communicated with. $\omega$ indicates the factor of path loss.

\section{COAlition Formation FOR CONSUMERS IN SMART GRID}

In this section, we formulate the cooperation of consumers for electricity purchase as a coalitional game $(\mathcal{N}, \mathcal{V})$ with transferable utility (TU). In this game, $\mathcal{N}$ is the set of consumers who seek to find partners in order to maximize their utilities. $\mathcal{V}$ is a mapping that determines the payoffs that these players receive in the game.

\section{A. Welfare Function}

For the $i$ th consumer, we represent its corresponding utility function as $\nu\left(P_{i, t}\right)$. The utility function represents the variation of consumers satisfaction changes with their power consumption. Generally, it is a kind of non-decreasing and concave function. The utility function adopted in this paper is shown as following [11],

$$
v\left(P_{i, t}\right)=\left\{\begin{array}{cc}
\delta P_{i, t}-\frac{\varepsilon}{2} P_{i, t}^{2} & 0 \leq P_{i, t} \leq \frac{\delta}{\varepsilon} \\
\frac{\delta}{\varepsilon} & P_{i, t}>\frac{\delta}{\varepsilon}
\end{array},\right.
$$

where $\delta$ and $\varepsilon$ are the pre-determined factor which may different among users and different times.

Therefore, if consumers cooperate with others and form a coalition, the welfare function of this coalition is

$$
V_{\mathcal{S}, t}\left(P_{\mathcal{S}, t}\right)=\sum_{i \in \mathcal{S}}\left[\nu\left(P_{i, t}-L_{i, \hat{i}}\right)\right]-C_{\mathcal{S}, t}\left(P_{\mathcal{S}, t}\right),
$$


where $P_{\mathcal{S}, t}=\sum_{i \in \mathcal{S}} P_{i, t}$ is the total power consumption in this coalition. $L_{i, \hat{i}}$ is the power cost for exchange information between consumer $i$ and the its farthest partner $\hat{i}$ in the same coalition. The cost in welfare function is the payment for power provider, that is $C_{\mathcal{S}, t}\left(P_{\mathcal{S}, t}\right)=P_{\mathcal{S}, t} p_{t}\left(P_{\mathcal{S}, t}\right)$, where the price function has already defined above.

\section{B. Coalition Formation Concepts}

Before we describe the coalition formation algorithm, some basic concept should be given [12].

Definition 1: (Network Structure) If the consumers in can be divided into disjoint coalitions $\mathcal{S}=\left\{\begin{array}{lll}S_{1} & \cdots & S_{M}\end{array}\right\}$, satisfies $\cup_{j=1}^{M} S_{j}=\mathcal{N}$ and $S_{j} \cap S_{k}=\phi, j \neq k, \mathcal{S}$ can be defined as a partition of $\mathcal{N}$ and indicates the network structure.

Definition 2: (Comparison Relation) Assume that $\mathcal{R}=$ $\left\{R_{1}, \cdots, R_{L}\right\}$ and $\mathcal{S}=\left\{S_{1}, \cdots, S_{M}\right\}$ are two different partitions of the same subset $\mathcal{A} \subseteq \mathcal{N}$. An operator $\triangleright$, such as $\mathcal{R} \triangleright \mathcal{S}$, is defined as the comparison relation between $\mathcal{R}$ and $\mathcal{S}$, which means that the way $\mathcal{R}$ partitions $\mathcal{A}$ is preferred to the way $\mathcal{S}$ partitions $\mathcal{A}$.

In well-known comparison relations, Pareto order is always in common use. It performs the comparison using the payoff of individual consumers.

Definition 3: (Pareto Order) Consider two partitions $\mathcal{R}=$ $\left\{R_{1}, \cdots, R_{L}\right\}$ and $\mathcal{S}=\left\{S_{1}, \cdots, S_{M}\right\}$. Define the utility of player $j$ in partitions $\mathcal{R}$ and $\mathcal{S}$ are $\phi_{j}(\mathcal{R})$ and $\phi_{j}(\mathcal{S})$. $\mathcal{R}$ is preferred over $\mathcal{S}$ by Pareto order if the following relationship is satisfied with at least one strict inequality for a player,

$$
\mathcal{S} \triangleright \mathcal{R} \Leftrightarrow\left\{\phi_{j}(\mathcal{S}) \geq \phi_{j}(\mathcal{R}), \forall j \in \mathcal{S}, \mathcal{R}\right\}
$$

In other words, if the network structure changes from $\mathcal{R}$ to $\mathcal{S}$, at least one player can obtain more payoff without hurting others benefit.

\section{The Modified Pareto Order}

In smart grid, in order to take full use of electricity resource, we define a new comparison relation, which not only compare the payoff of individual consumers as Pareto order, but also the social welfare.

Definition 4: (Modified Pareto Order) Consider two different partitions $\mathcal{R}=\left\{R_{1}, \cdots, R_{L}\right\}$ and $\mathcal{S}=\left\{S_{1}, \cdots, S_{M}\right\}$. The modified Pareto order denote that $\mathcal{R}$ is preferred over $\mathcal{S}$ if the following relationship is satisfied with at least one strict inequality for a player,

$\mathcal{S} \succ \mathcal{R} \Leftrightarrow\left\{\sum_{m=1}^{M} \mathcal{S} \geq \sum_{l=1}^{L} \mathcal{R} \mid \phi_{j}(\mathcal{S}) \geq \phi_{j}(\mathcal{R}), \forall j \in \mathcal{S}, \mathcal{R}\right\}$.

The modified Pareto order denotes that network structure $\mathcal{S}$ can bring more social welfare than $\mathcal{R}$ on the premise that the Pareto order can be maintained.
TABLE I. The CoAlition Formation Algorithm Based on the MODIFIED PARETO ORDER

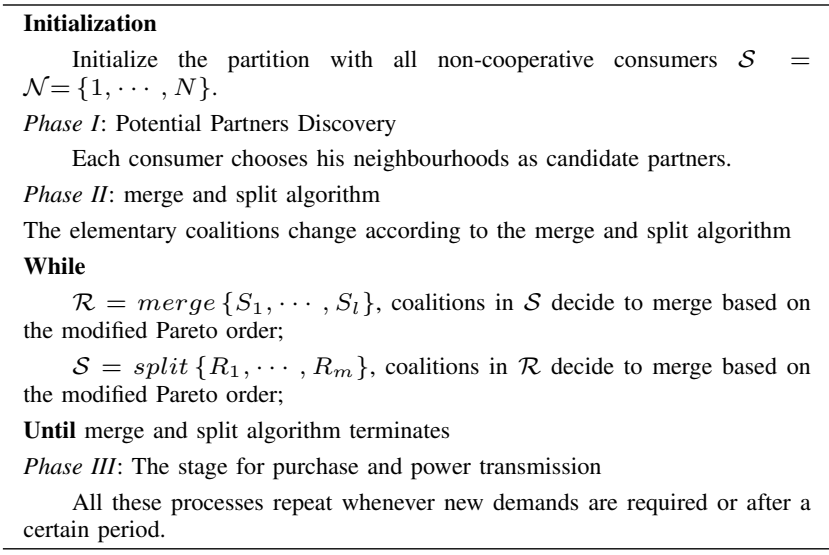

\section{Coalition Formation Algorithm}

Based on comparison relation defined before, we can devise the coalition formation algorithm for the self-organization of consumers in smart gird. This algorithm is based on two main rules named merge and split that allow to modify partitions [12]:

Merge Rule: For any set of coalitions $\left\{S_{1}, \cdots, S_{l}\right\}$, they can be merged whenever it is preferred by consumers, which can be expressed as $\left\{\bigcup_{j=1}^{l} S_{j}\right\} \succ\left\{S_{1}, \cdots, S_{l}\right\}$ (Operator $\triangleright$ instead when traditional comparison relation is used).

Split Rule: For any set of coalitions $\left\{S_{1}, \cdots, S_{l}\right\}$, they can be merged whenever it is preferred by consumers, which can be expressed as $\left\{S_{1}, \cdots, S_{l}\right\} \succ\left\{\bigcup_{j=1}^{l} S_{j}\right\}$ (Operator $\triangleright$ instead when traditional comparison relation is used).

According to these two rules, consumer coalitions decide to merge into less but larger coalitions or split to more but smaller coalitions whenever the behaviours gain more benefits. The detailed algorithm process is shown in TABLE. I.

The above three phases are repeated periodically during the operation time and is adaptive to the changes of consumers satisfaction which may be different in different time.

\section{Simulation Results}

To assess the performances of the proposed scheme, we set up the following simulations. For simplicity and without loss of generality, we deploy a $10 \mathrm{~km} 10 \mathrm{~km}$ square area as distribution network with a number of consumers distributed randomly. They are searching for partners to form coalitions. The parameters in the wholesale part of pricing model are setting as $\alpha=0.01$ and $\beta=\lambda=0$. In order to control the power load profile, the factor in price gap is chosen as. The target average power the power provider set to coalitions is $20 \mathrm{kw}$. We assume that the end-to-end SNR among consumers is about $10 \mathrm{~dB}$ and the target SNR requested by information exchange is $5 \mathrm{~dB}$. The path loss factor is 3 . The satisfaction factors $\delta$ may vary among different consumers and different time periods with central value 1 as simple. The maximum 


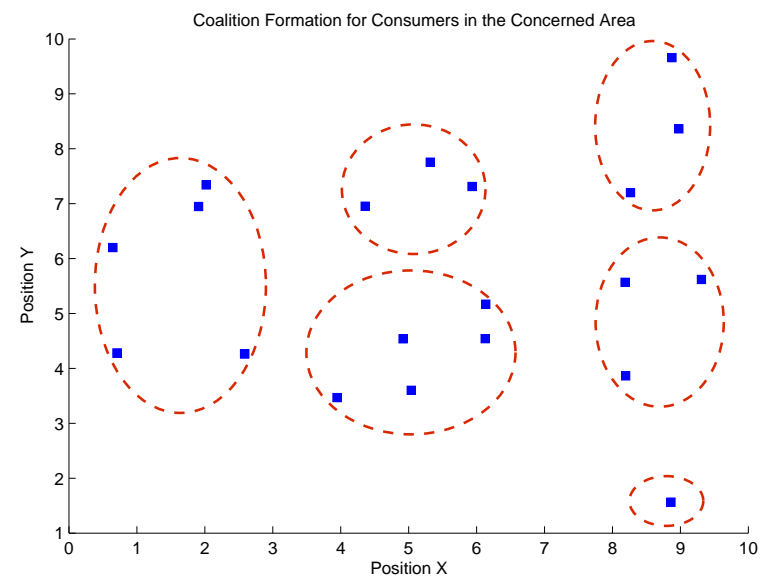

Fig. 2. The results of coalition formation in simulation scenario.

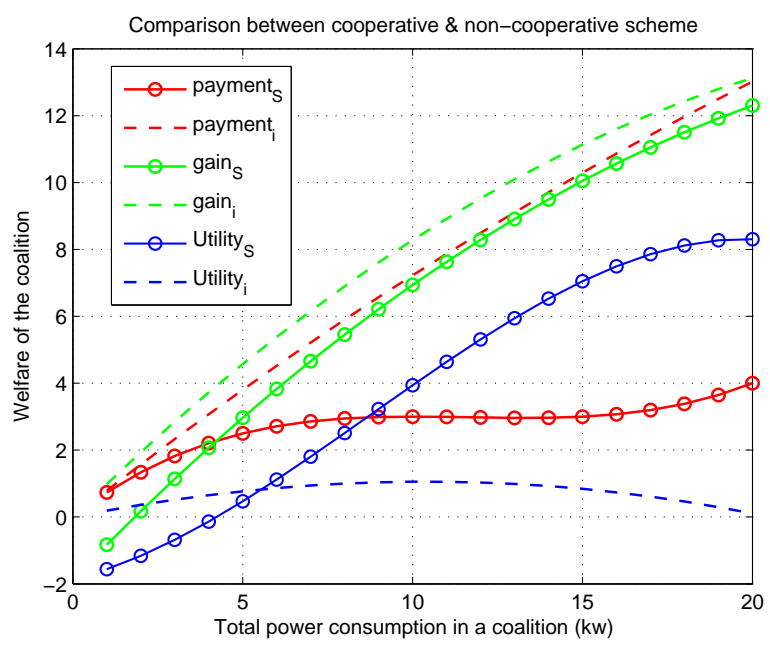

Fig. 3. The comparison between the coalition formation scheme and noncooperative scheme.

power consumption level of consumers are also different with each other.

Fig. 2 shows the results after coalition formation of the consumers in the concerned area. The scattered consumers keep searching their partners with merge and split rules until there is no more benefits can be got according to the modified Pareto order, and the process terminates. After the coalition formation process, several disjoint coalitions are established as shown in Figure 2. Since different consumers have different satisfaction for the same value of electricity, the radiuses and the number of consumers in coalitions are different. It is can be seen that the consumers who locate near with each other and have high satisfaction for electricity are more likely to cooperate together. It is not easy for the one who has low level satisfaction to participate in coalitions even it is near to someone in distance.

Fig. 3 shows the comparison between cooperative and non- cooperative scheme in term of the payment, gain and the final utility (total welfare) of a certain number of consumers. The dash lines indicate the results of the non-cooperative scheme. In this scheme, consumers pay for the power consumption separately. It can be seen that the total gains and the payment of all these consumers increase rapidly with the total power consumption of them, and therefore a small welfare value in general. The full lines with marks demonstrate the efficiency of coalition formation method. Though the total gains are lower than non-cooperative scheme due to the power cost in the process of coalition formation, the payment is saved which lead to a better welfare performance. However, if the amount of power consumption keep increasing, the welfare will decrease instead. Because the payment for the heavy power load will increase rapidly, and meanwhile the gains of all consumers will achieve saturation. Meanwhile, it can be seen that the value of welfare is lower than zero when the power consumption is low. The reason is that the payment saving in cooperation mode is negligible but the cost for coalition formation is very high. In these condition, non-cooperative method is better.

\section{CONCLUSION}

A cooperative power consumption scheme for consumers in smart grid is proposed in this paper. The two parts pricing model and the welfare function for coalition formation are suitable for general case in smart grid. For coalition formation, an algorithm based on the modified Pareto order is devised for consumers. Simulation results show the efficiency of the proposed scheme. A stable partition can be formed in this system. The proposed scheme can utilize the cooperative relationship among consumers for payment savings and meanwhile take the social welfare into consideration.

\section{ACKNOWLEDGMENT}

This work is partly supported by the National Science Foundation of China (Grant No. NFSC \#61071083, \#61371073) and the National High-Tech Research and Development Program of China (863 Program), No.2012AA01A506. Corresponding author: Jianjun Wu, E-mail: just@pku.edu.cn.

\section{REFERENCES}

[1] X. Fang, S. Misra, G. Xue, D. Yang, Smart Grid-The New and Improved Power Grid: A Survey, Communications Surveys \& Tutorials, IEEE, vol: 14, issue: 4, pp: 944 C 980, Fourth Quarter 2012.

[2] M. Alizadeh, X. Li ; Z. Wang ; A. Scaglione, R. Melton, "Demand-Side Management in the Smart Grid: Information Processing for the Power Switch”, IEEE Signal Processing Magazine, vol: 29, issue:5, pp: 55-67, Sept. 2012.

[3] A. Mohsenian-Rad, V. Wong, J. Jatskevich, R. Schober, A. LeonGarcia, Autonomous demand-side management based on game-theoretic energy consumption scheduling for the future smart grid, IEEE Transactions on Smart Grid, vol: 1, no. 3, pp. 320 C331, Dec. 2010.

[4] C. Ibars, M. Navarro, L. Giupponi, "Distributed Demand Management in Smart Grid with a Congestion Game", SmartGridComm, pp: 495-500, Oct. 2010.

[5] M. Neely, A. Tehrani, and A. Dimakis, Efficient algorithms for renewable energy allocation to delay tolerant consumers, SmartGridComm, pp: 549 C554, Oct. 2010. 
[6] M. Vinyals, F. Bistaffa, A. Farinelli, and A. Rogers, Stable coalition formation among energy consumers in the smart grid, in Proceedings of the 3rd International Workshop on Agent Technologies for Energy Systems (ATES), pp. 73-80, 2012.

[7] A. Yassine, "Analysis of a Cooperative and Coalition Formation Game Model among Energy Consumers in the Smart Grid", ICCIT, pp: 152 156, June 2013.

[8] Hung Khanh Nguyen, J.B. Song, Z. Han, Demand Side Management to Reduce Peak-to-Average Ratio using Game Theory in Smart Grid, INFOCOM WKSHPS, pp: 91-96, March 2012.

[9] Md Mahbubur Rahman Khan, Modeling of Consumer Responses to Dynamic Pricing in a Smart Grid, Master Thesis, North Dakota State University, Feb. 2012.

[10] M.W. Baidas, A.B. MacKenzie, On the Impact of Power Allocation on Coalition Formation in Cooperative Wireless Networks, WiMob, pp: 488-495, Oct. 2012.

[11] P. Samadi, A.-H. Mohsenian-Rad, R. Schober, V.W.S. Wong, Optimal Real time Pricing Algorithm Based on Utility Maximization for Smart Grid, SmartGridComm, pp:415-420, Oct. 2010.

[12] Z. Han, D. Niyato, W. Saad, Game Theory in Wireless and Communication Networks, ISBN: 9780521196963, Jan. 2012.

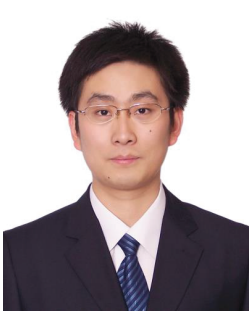

Xi Luan received his bachelor degree in electrical engineering from Tongji University, Shanghai, China, in 2008. Since 2008, he has been a PhD candidate in Institution of Advanced Communications, Peking University, China. His research interests are in the area of satellite mobile communications and wireless communications, such as MSS based on LTE, compressive sensing, cooperative communications. Email: luanxi@pku.edu.cn.

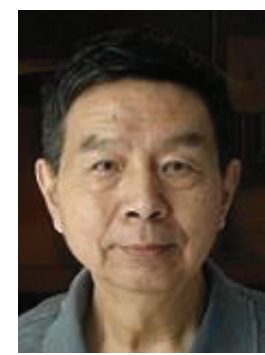

Haige Xiang received the bachelor of Engineering in Electronics from Peking University, Beijing, P. R. China, in 1964. Since 1964, he has been a professor at Electronics Department at Peking University. His research interests include information theory, wireless communications, channel coding, signal processing in communications and satellite communications. Professor Xiang is a member in IEEE. Email: xianghg@pku.edu.cn. 